\title{
Jürgen HABERMAS, 2012, La Constitution de l'Europe, Paris, Gallimard, « NRF Essais », 224 p.
}

\section{Pierre Étienne Vandamme}

\section{(2) OpenEdition}

12 Journals

Édition électronique

URL : http://journals.openedition.org/ress/2481

DOI : $10.4000 /$ ress.2481

ISSN : 1663-4446

Éditeur

Librairie Droz

\section{Édition imprimée}

Date de publication : 1 juin 2013

Pagination : 273-276

ISBN : 978-2-600-01749-7

ISSN : 0048-8046

\section{Référence électronique}

Pierre Étienne Vandamme, « Jürgen HABERMAS, 2012, La Constitution de l'Europe, Paris, Gallimard, "NRF Essais », 224 p. », Revue européenne des sciences sociales [En ligne], 51-1 | 2013, mis en ligne le 10 mai 2013, consulté le 22 septembre 2020. URL : http://journals.openedition.org/ress/2481 ; DOI : https://doi.org/10.4000/ress.2481

Ce document a été généré automatiquement le 22 septembre 2020.

(c) Librairie Droz 


\title{
Jürgen HABERMAS, 2012, La Constitution de l'Europe, Paris, Gallimard, « NRF Essais », 224 p.
}

\author{
Pierre Étienne Vandamme
}

1 Jamais sans doute un ouvrage de Jürgen Habermas n'aura autant « collé » à l'actualité européenne en l'occurrence. Non pas que l'Union européenne ait soudainement emprunté la voie idéale qu'il cherche à lui dessiner depuis quelque quinze années - loin de là -, mais plutôt parce que la crise à laquelle elle fait face témoigne avec une certaine évidence du «vice de construction » (p. 51) que Habermas - parmi d'autres - n'a cessé de dénoncer à son sujet. Presque tout le monde aujourd'hui, quelles que soient les orientations politiques, s'accorde à dire qu'une union monétaire sans union économique était vouée à l'échec, et que la voie de sortie de crise la moins coûteuse, désormais, semble être un pas en avant vers plus de fédéralisme, vers un transfert de souveraineté. Ceux qui ne situent les causes de cette crise que dans une réalité purement interne au «système» économique même sont aveugles, aux yeux de Habermas, et dissimulent ce faisant ses véritables enjeux, qui sont politiques.

2 Cependant, le nécessaire progrès dans la constitution progressive de l'UE ne trouve pas sa justification, aux yeux de Habermas, dans la logique du moindre coût (qui justifierait le renforcement de la zone euro par rejet du coût de sa dissolution), mais dans la dynamique « civilisatrice » (au sens allemand, moins connoté négativement, du terme) de la progressive constitutionnalisation internationale du droit. Au cœur de cette dynamique réside la tension (féconde, du point de vue des relations internationales) entre la portée universaliste des droits de l'homme et leur réduction aux droits des citoyens. À cet égard, Habermas fustige sans concession la redécouverte par l'Allemagne d'Angela Merkel d'un égoïsme national et d'une nouvelle fierté liés à sa remarquable santé économique, avec cette conséquence que «le gouvernement fédéral allemand est devenu un accélérateur de désolidarisation à l'échelle européenne » (p. 69). 
3 Mais outre ce déclin de la solidarité, c'est celui de la démocratie qu'il faut craindre, à l'en croire, le déficit démocratique de l'UE ne faisant que s'aggraver à mesure que se succèdent les interventions technocratiques visant à retrouver l'équilibre économique perdu. Cependant, si la méthode intergouvernementale n'a pas les faveurs de Habermas, la justification de ce rejet demeure quelque peu ambiguë. « Le "pacte pour l'euro", dit Habermas, perpétue une vieille erreur : non contraignants juridiquement, les accords passés en petit comité entre les chefs d'État et / ou de gouvernement sont soit inefficients, soit non-démocratiques ; il faut, par conséquent, les remplacer par une institutionnalisation des décisions communes qui soit démocratiquement irréprochable. » (p. 69) Il semble que Habermas élude, ce faisant, une probable tension entre justice et démocratie. En effet, d'une part, on peut aussi bien imaginer des accords efficients mais non démocratiques, et de l'autre une procédure "démocratiquement irréprochable» débouchant sur des résultats largement insatisfaisants eu égard aux attentes normatives de Habermas. Dès lors, si ce dernier a raison de stigmatiser la gestion technocratique de l'UE, il devrait également souligner le danger qu'il y aurait à soumettre entièrement, à l'heure actuelle, le sort du projet européen à la volonté de ses peuples.

Cette tension entre justice sociale et démocratie est au cœur de l'œuvre de Habermas. Or, la crise actuelle de l'UE concerne ces deux dimensions. À ce sujet, le philosophe allemand précise que la " formation commune de la volonté démocratique » (p. 106) audelà de la nation implique une « solidarité civique élargie » (ibid.), qui déborde elle aussi du cadre national. La solidarité apparaît dès lors, à ses yeux, comme condition de possibilité de l'agir collectif démocratique. Mais quel degré de solidarité ? Habermas s'en réfère à l'idée contenue dans la Loi fondamentale de la République fédérale allemande, selon laquelle il convient de préserver "l'homogénéité des conditions de vie » (art. 106, al. 3) entre les diverses entités fédérées. Bien interprété, au niveau européen, ce principe devrait impliquer des redistributions importantes - une forme d'euro-dividende (Van Parijs) ? -, au sujet desquelles il demeure, hélas, muet.

Les vues de Habermas, dans cet ouvrage, vont cependant bien au-delà de l'actualité immédiate de l'UE, puisqu'elles inscrivent celle-ci dans la constitutionnalisation internationale du droit. À ce propos, le philosophe affronte les réticences habituelles. La démocratie est-elle seulement possible au-delà de la nation? Oui, répond-il sans hésiter. Car comment se définit la démocratie? Par la capacité qu'a une communauté politique d'agir sur elle-même. "L'action coopérative des citoyens sur les conditions sociales de leur existence ne devient cependant effective que si un État dispose d'une latitude d'action qui puisse répercuter cette action coopérative dans l'aménagement politique des conditions de vie.» (p. 79) Or, puisque les États nationaux ont désormais les mains liées du fait de la mondialisation, c'est la sauvegarde même de la démocratie qui justifie son changement d'échelle ; « il y va du sens normatif de la démocratie ellemême que les capacités politiques d'action puissent être étendues au-delà des frontières nationales » (p. 79).

6 Mais quelle forme devrait alors revêtir cette démocratie supranationale? Dans la recherche par Habermas d'une voie médiane entre l'idéal de l'État fédéral et le compromis de la fédération d'états, on retrouve le même débat qui était déjà au cœur du Projet de paix perpétuelle de Kant, et qui est également sous-jacent au problème de Rawls concernant la justice mondiale, celui de la tension entre droit cosmopolitique et droit des peuples. Chez Rawls, ce sont les représentants des peuples qui sont placés 
sous voile d'ignorance international, au détriment des individus. Chez Kant, à en croire Claude Piché, l'État fédéral mondial demeure l'idéal théorique, bien qu'un compromis pragmatique incline à lui préférer (à moins long terme ?) la fédération des États du monde. Habermas, pour sa part, cherche à bâtir un modèle intermédiaire qui «équilibre » (p. 119) les prérogatives individuelles des citoyens du monde et celles des États. Cependant, la justification principale qu'il fournit est également celle d'un compromis pragmatique : l'analyse du réel incline à prendre en compte les résistances à l'abandon de souveraineté de la part des États-nations, du fait de l'importance historique de ces derniers - ce qui ne fournit pas de défense normative du maintien de la souveraineté nationale. Il semble donc y avoir, de ce point de vue, une certaine confusion des ordres descriptif et normatif, au détriment de l'idéal régulateur.

7 Cependant, on peut trouver ailleurs (p.114) dans l'essai de Habermas une autre justification de l'abandon du modèle de l'État fédéral pour ce qui concerne, à tout le moins, l'Union européenne. Bien que la forme hypothétique de l'État fédéral européen soit sans doute la plus à même, théoriquement, de satisfaire les réquisits normatifs des droits de l'homme (économiques et sociaux, en particulier), la forme intermédiaire entre État fédéral et fédération d'États («Union constitutionnelle»?) préservant le monopole de la force légitime pour les États tout en les soumettant au droit supranational, possède cet avantage d'être plus ouverte que la première. En effet, si le projet européen est à entendre dans un sens cosmopolitique, il est probable que cette forme intermédiaire soit la plus à même de se répandre pour former, à l'horizon utopique, une Union des peuples et des citoyens du monde, sans qu'il soit besoin pour cela de construire une telle identité commune qu'elle permette la fusion des diverses nations dans un État mondial intégré, fût-il fédéral.

8 Se pose alors une dernière question. L'ordre mondial imaginé par Habermas, où le ressort d'action de l'Organisation mondiale serait «plus juridique que politique » (p. 125), se cantonnant au maintient de la paix et au respect des droits de l'homme, serait-il capable de véritable justice distributive globale? Comment s'assurer réellement du respect des droits socio-économiques tels que le droit au travail et le droit à un salaire égal pour un travail égal (art. 23 de la Déclaration universelle)? Cela requiert certainement davantage, d'un point de vue institutionnel, que ce qu'imagine Habermas, sans quoi on en demeurerait, au niveau global, à ce droit bourgeois d'avant la critique marxiste des droits de l'homme, méconnaissant les effets de la structure de base mondiale sur la liberté réelle des citoyens du monde.

Habermas reconnaît toutefois ce problème et se justifie en affirmant que si les inégalités mondiales sont bel et bien intolérables, il faut admettre que l'homogénéisation planétaire des conditions de vie demeure un horizon largement utopique et que, dans un premier temps, tout ce qu'on peut espérer est l'instauration d'une démocratie mondiale, fût-elle extrêmement inégalitaire. Cependant, ajoute-t-il, on peut également espérer que le débat public mondial mène, à long terme, à « une harmonisation effective et politiquement voulue des conditions de vie » (p. 130).

Il se livre alors, pour conclure, à une généalogie de l'intrication du corpus « indivisible» (p. 141) des droits de l'homme et du concept de dignité humaine, coaffirmés dans l'incipit de la Déclaration universelle. De la compréhension antique, exclusive, de la dignité, à l'universalisation kantienne, en passant par l'égalitarisme judéo-chrétien, cette émergence conjointe est ancrée dans une histoire particulière. Pourtant, remarque-t-il, il se produit autour de cette notion de dignité humaine comme 
un « consensus par recoupement » (p. 138), selon l'expression rawlsienne ; chacun peut s'y reconnaitre à partir de son contexte culturel propre. Or, cette affirmation, au cœur de nos systèmes moraux et juridiques, nous enjoint à ne jamais nous satisfaire d'une restriction des droits de l'homme aux droits des citoyens. Puisque c'est l'humanité en l'autre qui lui confère sa dignité et ses droits, c'est au niveau d'une Constitution mondiale qu'il s'agit d'institutionnaliser les droits de l'homme, fût-ce au détriment d'un peu de souveraineté étatique. Pour ce faire, il convient de se frayer un passage entre une pure rhétorique des droits de l'homme qui cache un impérialisme et des intérêts non universalisables (p.155-156), d'une part, et une réduction des droits de l'homme à leur valeur politique pour une société déterminée (p. 156-157), d'autre part. Conserver leur contenu moral universaliste est le seul moyen, conclut Habermas, d'honorer le lien interne et indéfectible entre ces droits et l'égale dignité de tous les humains.

\section{AUTEURS}

\section{PIERRE ÉTIENNE VANDAMME}

Université catholique de Louvain 